\title{
Stem cell activation during hair regeneration
}

\author{
Valentina Greco, H. Amalia Pasolli, Ting Chen and Elaine Fuchs.
}

Howard Hughes Medical Institute, Laboratory of Mammalian Cell Biology and

Development, The Rockefeller University, 1230 York Avenue, New York, NY 10065.

Adult stem cells (SCs) function in tissue homeostasis and wound repair. In the adult skin, slow-cycling, relatively undifferentiated SCs exist within a niche known as the bulge, located below the sebaceous gland in the outer root sheath of the hair follicle (HF). Hair follicles undergo cycles of growth (anagen), destruction (catagen) and rest (telogen).

Many questions remain in understanding how SCs become activated at the start of each new hair cycle (1). To address these questions, we have focused on two subcompartments of the lower follicle that exist throughout telogen: the CD34 $(+)$, slow cycling bulge cells and the (secondary) hair germ (HG), a small cluster of P-cadherin-enriched cells that form at the end of catagen and separates bulge from underlying dermal papilla $(1,2)$.

We showed that the HG maintains its size, shape and morphology throughout telogen, where it persists as an ultrastructurally distinct entity. Our studies support the bulge as precursor of the HG. We uncover a two-step activation process: HG cells proliferate several days before bulge cells and are the major contributors to the first stage in HF regeneration.

Transcriptional profiling reveals that during telogen, both HG and DP are more dynamic than bulge cells. Key signaling proteins required for the telogen to anagen transition accumulate in these compartments in late telogen, with Wnt signaling in HG and elevated FGFs and BMP inhibitors in DP. These findings provide significant new insights into how stem cell activation is orchestrated in the hair follicle. Our findings suggest a model where HG cells fuel initial steps in hair regeneration, while the bulge is the engine maintaining the process.

[1] Greco V, Chen T, Rendl M, Schober M, Pasolli HA, Stokes N, delaCruz-Racelis J, and Fuchs E. A two-step mechanism for stem cell activation during hair regeneration. Cell Stem Cell 4, 155-169, February 6, 2009.

[2] Blanpain C, Lowry WE, Geoghegan A, Polak L, Fuchs E. Self-renewal, multipotency, and the existence of two cell populations within an epithelial stem cell niche.

Cell, 118(5):635-48. Sep 32004. 


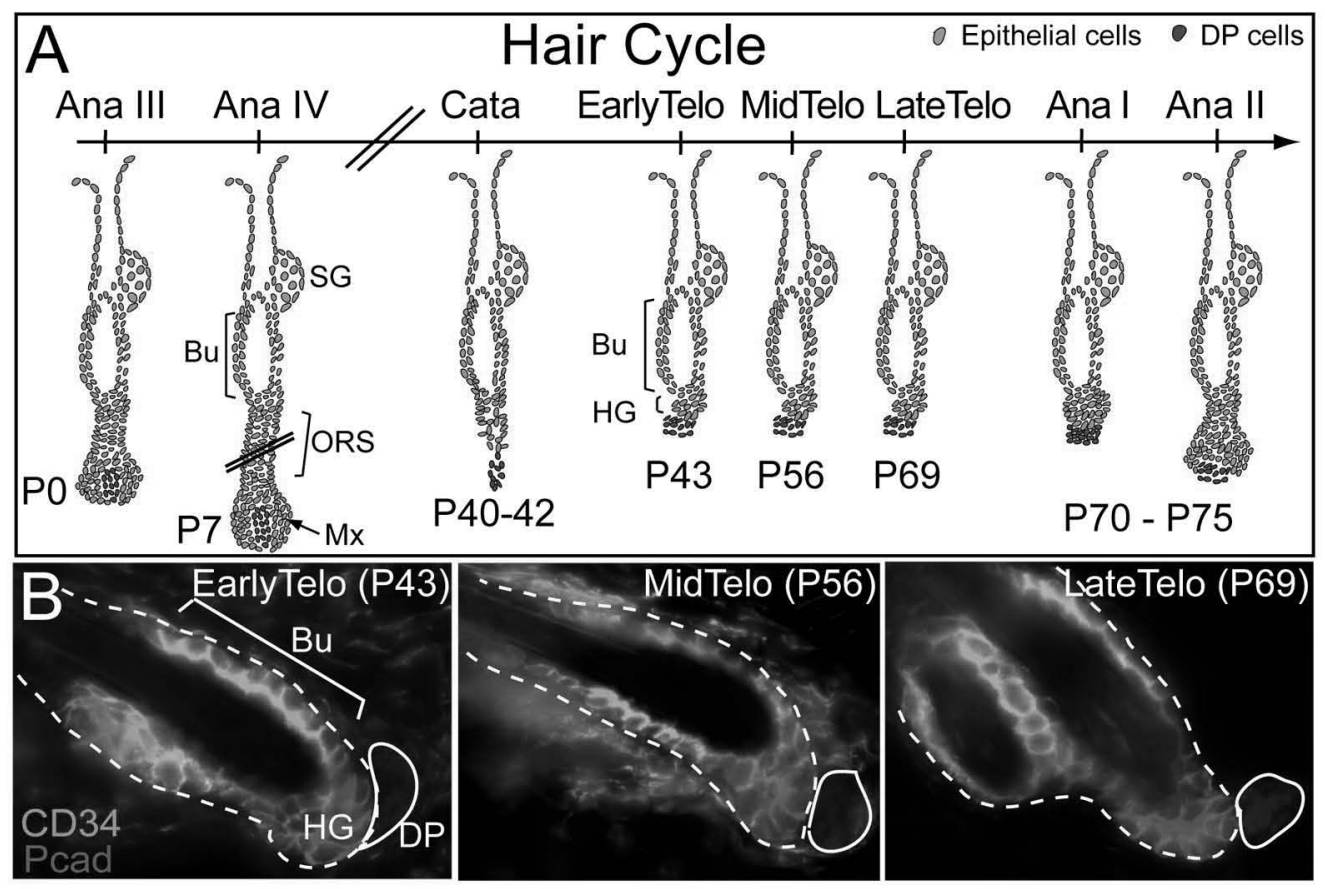

Figure 1. (A) Schematic of the hair cycle. The hair germ (HG) emerges at the end of the first catagen and exists as a distinct entity during telogen. Abbreviations: $\mathrm{Bu}$, bulge; SG, sebaceous gland; ORS, outer root sheath; Mx, matrix. (B) The HG and bulge are distinguished by immunofluorescence for enrichment of P-cadherin (Pcad, HG) and CD34 (Bu) and by its close proximity to the dermal papilla (DP), denoted by the solid white line.

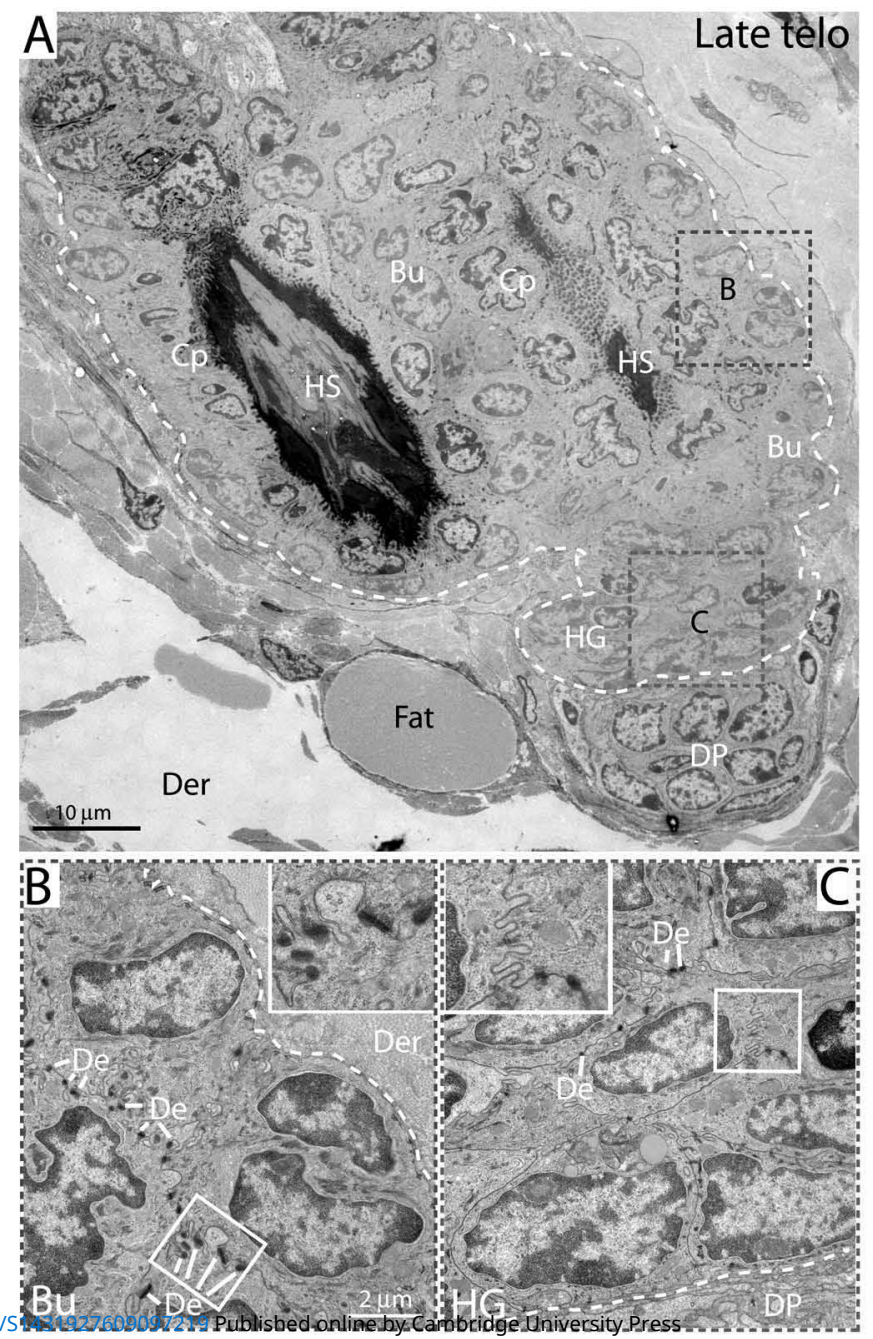

Figure 2. Ultrastructural analyses reveal morphological distinctions between HG and bulge cells. HG cells display higher cytoplasmic density but possess fewer desmosomes (De). (A) Ultrastructure of hair follicle in late telogen. Bulge $(\mathrm{Bu})$ and hair germ $(\mathrm{HG})$ cells are highlighted in green and pink, respectively. Dotted line denotes the presence of the basement membrane. $\mathrm{Cp}$, companion layer contiguous to bulge cells. DP, dermal papilla; HS, hair shaft; Der, dermis. (B) Bulge cells display numerous desmosomes (De), especially in their attachment to the companion layer. Boxed area enlarged in inset, showing intercellular junctions rich in desmosomes. (C) Hair germ cells possess fewer desmosomes. Boxed area is magnified in inset, showing intercellular junctions with scarcity of desmosomes. Both figures, figure legends and part of abstract reprinted from reference (1) with permission from Elsevier. 